Article

\title{
The Sequential and Contractible Topological Embeddings of Functional Groups
}

\author{
Susmit Bagchi \\ Department of Aerospace and Software Engineering (Informatics), Gyeongsang National University, \\ Jinju 660701, Korea; profsbagchi@gmail.com
}

Received: 9 April 2020; Accepted: 7 May 2020; Published: 8 May 2020

\begin{abstract}
The continuous and injective embeddings of closed curves in Hausdorff topological spaces maintain isometry in subspaces generating components. An embedding of a circle group within a topological space creates isometric subspace with rotational symmetry. This paper introduces the generalized algebraic construction of functional groups and its topological embeddings into normal spaces maintaining homeomorphism of functional groups. The proposed algebraic construction of functional groups maintains homeomorphism to rotationally symmetric circle groups. The embeddings of functional groups are constructed in a sequence in the normal topological spaces. First, the topological decomposition and associated embeddings of a generalized group algebraic structure in the lower dimensional space is presented. It is shown that the one-point compactification property of topological space containing the decomposed group embeddings can be identified. Second, the sequential topological embeddings of functional groups are formulated. The proposed sequential embeddings follow Schoenflies property within the normal topological space. The preservation of homeomorphism between disjoint functional group embeddings under Banach-type contraction is analyzed taking into consideration that the underlying topological space is Hausdorff and the embeddings are in a monotone class. It is shown that components in a monotone class of isometry are not separable, whereas the multiple disjoint monotone class of embeddings are separable. A comparative analysis of the proposed concepts and formulations with respect to the existing structures is included in the paper.
\end{abstract}

Keywords: topological spaces; group decomposition; homeomorphism; sequence; Schoenflies embeddings

MSC: 54E05; 54E15; 54H10

\section{Introduction}

The continuous and injective embeddings in topological spaces have wide varieties as well as respective applications. In general, the topological embeddings consider connected spaces, which are at least first countable in nature. In a first countable $T_{2}$ space, the embeddings can generate closed subspaces with paracompactness. Interestingly, in the case of elementary type embeddings, a partial ordered set can be completely embedded into the space of an inner model [1]. The various categories of topological embeddings and associated homeomorphisms give rise to a set of interesting and insightful properties. For example, the Haefliger type embeddings of topological manifolds show that there exist homotopy equivalences with commutativity, and the locally flat embedded discs are generated in a bounded manifold (i.e., manifold with boundary) [2]. The topological embeddings of decomposed groups also have a wide array of applications [3,4]. The traditional group decomposition structures are based on the existence of independent subgroups, and as a result the semidirect product-based decompositions are not unique [5]. In case of block-based direct decomposition, the identity element plays a key role in generating independent modules [6]. However, the decomposition of groups in 
regard to topology is an interesting approach due to the enhanced flexibility and generality in the decomposed structures. In general, the topological embeddings of decomposed structures can be placed in two categories. From the algebraic viewpoint, the embedding of algebra $G$ into algebra $G^{1}$ is a linear homeomorphism [7]. On the other hand, the topological embedding in a metrizable space (i.e., Hausdorff space) is an isometry, which can be supercompact with a large cardinal [1]. This paper proposes the generalized algebraic formulation of functional groups and their sequential, as well as contractible embeddings in normal topological spaces. First, a brief description of various aspects of topological embeddings is presented in order to establish elementary concepts. In addition, the research questions dealt in this paper and the motivational aspects are presented.

\subsection{Topological Embeddings}

There exists a hierarchy of topological spaces considering the interplay of Borel and Hausdorff properties [8]. The Borel and Hausdorff hierarchies of topological spaces are based on axiomatic set theory and they are placed in the Polish space category [9]. The property of such topological spaces is that they are homeomorphic to the complete metric spaces. Moreover, such spaces are separable, because they are composed of countable dense sets. Furthermore, the Hausdorff topological spaces can be classified based on associated closure properties in normal topological spaces. The normal topological spaces can be of two varieties, namely completely normal and fully normal [9]. Moreover, the H-closure based classification of spaces is dependent on the concept of a strongly closed graph. For example, if $\omega$ is a class of topological spaces and $\forall X \in \omega, \exists Y \in \omega$ such that $f: X \rightarrow Y$ is a strongly closed function with weak continuity, then $\left(Y, \tau_{Y}\right)$ is a H-closed topological space [8,10]. In general, if the embeddings are compact in a topological space, then there exist closed subspaces within the respective topological space.

It is interesting to note that, topological embeddings of a continuous map in higher dimensional manifolds are not always a straight-forward approach, and there exist obstructions if the manifolds are differentiable in type [11]. The embeddings of spaces, functions, or groups within a topological space often maintain local homeomorphism within the space. Following a similar approach, the Schoenflies embeddings are often considered to be homeomorphic to $S^{1}$, and as a result it generates separable components [12]. In the case of the higher dimensional manifold, the continuum embeddings of a space within the manifold may generate at most two separable components [13]. The Jordan curve theorem (JCT) represents that a simple closed curve generates multiple separable components in a topological space [14-16]. The interesting property of JCT is that one of the components is bounded and possibly compact. However, the exterior of the closed component may not be compact if the topological space itself is not compact.

\subsection{Motivation}

Topological decompositions of group algebraic structures have varieties, and are an interesting topic with potential applications $[3,17]$. The topological decomposition of general group structures and associated embeddings in topological spaces are relatively new approaches without emphasizing the continuity criteria in group structures [18]. Alternatively, the concept of functionally generated groups attempts to incorporate continuity within the finite group structure. Interestingly, the functionally generated groups have various computational applications. For example, the groups generated by round functions form a set of cryptographic block ciphers [19]. There are various ways to form functionally generated groups with different characterizations. The circle group is a functionally generated group that has subgroups with sequential characterization [20]. The circle group on the Gauss plane is homeomorphic to $S^{1}$ and can be considered as a special function group, which is closed as well as embeddable in topological spaces. However, the interesting question is how to algebraically construct a generalized functional group structure and how to analyze the sequential embeddings of such structures in topological spaces. Moreover, if the topological embeddings are sequentially characterized along with contraction, then what are the properties of such an embedding sequence 
in a normal topological space? This paper addresses these questions in regard to general topology and analysis. First, the basic concept of topological decomposability of a general group structure is presented. Next, the algebraic construction of a generalized functional group structure and the sequence of embeddings in topological spaces are presented. The inherent properties of such a sequence of embeddings in normal topological spaces are explained as a set of theorems. The preservation of homeomorphism under contraction in a Hausdorff topological space is analyzed in regard to sequential topological embeddings inline to Schoenflies variety.

The rest of the paper is organized as follows. Section 2 presents preliminary concepts related to groups, Jordan curve theorems and morphisms. Section 3 describes concepts and definitions of generalized functional groups, decomposition structures and contractible sequential embeddings in topological spaces. The analytical properties are presented in Section 4 as main results in the form of theorems. Section 5 presents comparative analysis. Finally, Section 6 concludes the paper.

\section{Preliminary Concepts}

In this section, a set of basic concepts and definitions are presented to establish preliminary notions about topological embeddings, groups and related analysis. In this paper, $\bar{A}$ denotes the closure of the corresponding open set $A$, and the complement of set $A$ is given by $A^{c}$. Furthermore, it is considered that the normal topological space has a Hausdorff closure property, which may contain fixed points [10]. The power set of any arbitrary set $X$ is denoted by $P(X)$. The symbol $A \triangleleft B$ denotes that, $A$ is a subgroup of $B$.

Let $X$ be a set and $\left(X, \tau_{X}\right)$ be a topological space, which is considered to be Hausdorff to avoid multiconvergence localities. In a normal and complete Hausdorff topological space, the bijection $f_{B}: X \rightarrow X$ contains a set of fixed points given by, $X_{F}=\left\{x \in X: f_{B}(x)=x\right\}$. Let $X$ be equipped with abstract algebraic operation $*: X^{2} \rightarrow X$ such that the operation is closed in the set. The structure $G=(X, *)$ is called a group if it maintains the properties [9,21], (1) $\forall a, b, c \in X,(a * b) * c=a *(b * c)$, (2) $\forall a \in X, \exists a^{-1} \in X, a * a^{-1}=a^{-1} * a=e \in X$ and, (3) $\forall a \in X, e * a=a * e=a$. The element $e \in X$ is unique and $e * e=e$ indicates that $e=e^{-1}$.

A group $G=(X, *)$ can be equipped with a topological structure. The topology on $G=(X, *)$ is denoted by $\tau_{G} \subseteq P(X)$, where it maintains the axioms of topology. A group action is given by $\beta: G \times S \rightarrow S$ under specific conditions, where $S$ is a set on which a group is acted on. The required properties related to group action are (i) $\beta(g, s)=g * s$, and (ii) $\beta(g, \beta(h, s))=\beta((g * h), s)$. The second property asserts associativity of $G=(X, *)$ in the presence of group action. The identity function is given by id : $A \rightarrow A$ such that $i d(a)=a \in A$ in any arbitrary set $A$. If $G=(X, *)$ is a groupoid, then the morphism is given by $\alpha:(x \in G) \rightarrow(y \in G)$ in the groupoid [22]. The morphisms $\alpha_{1}, \alpha_{2}, \alpha_{3}$ maintain associative composition law given by $\alpha_{1} \circ\left(\alpha_{2} \circ \alpha_{3}\right)=\left(\alpha_{1} \circ \alpha_{2}\right) \circ \alpha_{3}$.

A topological group is a variety of groups in a topological space such that continuity of space under the closed algebraic operation within the space is maintained. The structure $\left(X, *, \tau_{X}\right)$ is a topological group if it maintains the axioms [23], (a) $G=(X, *)$ is a group, (b) $\left(X, *, \tau_{X}\right)$ is a topological space, and (c) the algebraic operation $*: X^{2} \rightarrow X$ is continuous along with the continuous existence of $a^{-1} \in X$. Furthermore, if $a, x \in X$ in $\left(X, *, \tau_{X}\right)$ are two elements and $a$ is the fixed element, then the transformation $T(x)=a x$ is a homeomorphism of $\left(X, *, \tau_{X}\right)$ into itself. A topological group $G=(X, *)$ can be compactly generated if $H \triangleleft G$ and $\exists Y \subseteq X$ such that $H=(Y, *)$ is a subgroup in compact subspace [9].

The Jordan curve $\Gamma$ in a topological space $\left(X, \tau_{X}\right)$ representing a plane, is a function $\gamma: C \rightarrow X^{2}$, where $C \subset \mathcal{R}^{2}$ is a closed curve in 2-D real such that $\gamma($.$) is continuous and injective [14]. It is to note$ that $C \subset \mathcal{R}^{2}$ can be any polygon in space and $C$ homeomorphic to $S^{1}$ can be considered as a circle group. There can be embeddings of closed curves in surfaces leading to the Jordan curve theorem (JCT) [24]. The embedding of $C \subset \mathcal{R}^{2}$ in a surface $S_{S}$ is called 2-cell embedding if all the regions are 2-cells [15]. Interestingly, in group algebra, the semigroups can be embedded within the group structures, which is actually not a topological embedding. However, the formulation of embedding in a group is not a 
straight-forward approach. The Steinitz embedding theorem shows that every integral domain can be embedded in a field [16]. The Steinitz theorem relies on the construction of ordered pairs of elements maintaining an equivalence relation.

\section{Decomposition and Functional Groups Embeddings: Concepts and Definitions}

This section presents a set of definitions related to topological decomposition as well as embeddings of general groups, the concept of functional groups and the related varieties of embeddings into normal topological spaces. In this paper, if $A$ is a set, then $A^{o}$ will denote the interior of the open set $A$ in the corresponding topological space, where $\bar{A}$ is a closed set as mentioned earlier. For any point $x \in A$ in topological space, the open set $U_{x}$ denotes an open neighborhood of the corresponding point. In this paper, the subset $I \subset Z_{0}^{+}$is employed as an index set and $\mathcal{R}$ represents the set of real numbers. The notation $\operatorname{hom}(A, B)$ denotes that $A$ is homeomorphic to $B$. The definitions are categorized into two sections. First, a set of definitions related to the topological decomposition of general group algebraic structure and associated decomposed group embeddings are described. Next, the definitions related to algebraic formulation of functional groups and their sequential embeddings into normal topological spaces are presented.

\subsection{Embeddable Topological Decomposition of Groups}

In this section, the topological decomposition and embeddings of a general group algebraic structure in the lower dimension is presented. It is important to note that the proposed construction does not assume any specific topological group structure. Essentially, a topological group is a group residing in topological spaces, where the group operations and actions are continuous in nature [23]. However, in this proposed formulation of decomposition, it is considered that a standard group can be decomposed in view of general topology and the decomposed components are embeddable in normal topological spaces. Thus, if $G=(X, *)$ is a group, then $\left(G, \tau_{G}\right)$ represents an embedded group structure in normal topological spaces, where $\tau_{G} \subseteq P(X)$ and $\beta: G \times S \rightarrow S$ is a group action on some arbitrary set $S$.

First, the notion of the partition of a normal topological space is established as follows. Let $\left(X, \tau_{X}\right)$ be a topological space and the partition of the space is given by $\Pi_{X}=\left\{A_{i} \subset X: i \in I\right\}$, which is a family of subspaces [18]. It maintains the property as given below:

$$
\begin{aligned}
& \forall A_{i}, A_{j} \in \prod_{X}, A_{i} \cap A_{j}=\phi, \\
& \cup i \in I \\
& \forall A_{i}=X .
\end{aligned}
$$

A homogeneous set can be derived from a partitioned topological space [25]. The partitioning of the topological space based on the generation of a quotient set containing equivalence classes may not affect separability of the original space. Let $G_{1}=\left(X_{1}, *_{1}\right)$ and $G_{2}=\left(X_{2}, *_{2}\right)$ be two groups, which are embeddable in a normal topological space. The group homeomorphism is given by $h_{H}: G_{1} \rightarrow G_{2}$ such that $\forall x, y \in G_{1}, h_{H}\left(x *_{1} y\right)=h_{H}(x) *_{2} h_{H}(y)$. Interestingly, the group homeomorphisms can be strengthened to form isotropy between group structures [26]. Let $\left(X, \tau_{X}\right)$ and $\left(Y, \tau_{Y}\right)$ be two topological spaces and $F:(A \subset X) \rightarrow Y$ be an embedding, which is continuous as well as injective in nature. There exists a Schoenflies homeomorphism $h_{S}: X \rightarrow Y$ such that the restriction maintains $\left.h_{S}\right|_{A \subset X}=F$. The Schoenflies homeomorphism may generate multiple separable components in topological spaces if the embedded curve is a closed type [12].

Next, a set of definitions related to embeddable topological decomposition of group algebraic structure is presented [18]. 


\subsubsection{Definition of $G-$ Partition}

Let $D_{G} \subset \tau_{G}$ be in the normal topological space such that $i, k \in I, i \neq k$ and the following structure is maintained by it:

$$
D_{G}=\left\{A_{i} \subset X: A_{i} \in \tau_{G} \wedge\left(A_{i} \cap A_{k}=\phi\right)\right\} .
$$

The $G$ - partition of $\left(G, \tau_{G}\right)$ is denoted by $D_{G}$, which generates a family of mutually disjoint open sets representing subspaces in the corresponding normal topological space.

\subsubsection{Topological Decomposition and Fiber}

A topological decomposition of $G=(X, *)$ resulting in the formation of $\left(G, \tau_{G}\right)$ is denoted by $\prod_{G} \subset D_{G}$ if the following axioms are satisfied considering $A \in \tau_{G}, B \in \tau_{G}$ and $A \cap B=\phi$,

$$
\begin{aligned}
& X \backslash\{e\}=A \cup B, \\
& \forall a \in A, \exists b \in B: a * b=b * a=e \in G, \\
& \prod_{G}=\{A, B, X \backslash \overline{A \cup B}\} .
\end{aligned}
$$

The generalized decomposition structure prepares partitioned open sets within a topological subspace, where the subspace is finite. Let $f: A \rightarrow B$ be a bijection in the $\prod_{G}$. The function $f($.$) is a$ $\Pi_{G}-$ fiber if the following axiom is satisfied,

$$
\forall a \in A, \exists b \in B: a * f(a)=b * f^{-1}(b)=e .
$$

The concept of topological fiber enables relational mapping within partitioned subspaces within a topological space.

Remark 1. Interestingly, the topological one-point compactification property can be applied to the Gdecomposition to prepare a compact subspace. Let $\left(Y, \tau_{Y}\right)$ be a normal topological space such that $X \subset Y$ and $\tau_{X}=\left\{X \cap E: E \in \tau_{Y}\right\}$ generating $\left(X, \tau_{X}\right)$ as a topological subspace. As $A \subset X, B \subset X$ so $\{A, B\} \subset \tau_{X}$ indicates $A \cup B$ is open in $\left(Y, \tau_{Y}\right)$. Moreover, in $\left(X, \tau_{X}\right)$ it is true that, $X \backslash(A \cup B) \neq \phi$. Thus, if $\exists\{e\} \subset X$ such that $X \backslash(A \cup B)=\{e\}$ and $\left(X, \tau_{X}\right)$ is a compact topological subspace, then $G$-decomposition induces one-point compactification of $\left(X, \tau_{X}\right)$ by $\{e\}$.

The generalized Schoenflies embeddings and associated homeomorphism concepts are now applied to the decomposed group to form the decomposed embeddings in normal topological spaces, which results in the following definition.

\subsubsection{Decomposed Group Embedding}

Let $\left(X, \tau_{X}\right)$ be a normal topological space and $E \in \tau_{X}$ be a subspace. If $G=(Y, *)$ is a group, then the decomposed group embedding in $E$ is given by

$$
\begin{aligned}
& i, k \in Z^{+}, i, k \in[1,3], \\
& \exists H_{i} \subset E, D \in \prod_{G}, \\
& e_{i}: D \rightarrow H_{i}, \\
& {[i \neq k] \Rightarrow\left[H_{i} \cap H_{k}=\phi\right] .}
\end{aligned}
$$

Note that, embedding $e_{i}: D \rightarrow H_{i}$ is injective in nature within a normal topological subspace. It further concludes that $\underset{i \in[1,3]}{\cup} H_{i} \subset E$ in the topological space $\left(X, \tau_{X}\right)$. Note that the strict condition of homeomorphism to $S^{1}$ of an embedding is not applied in this case to maintain generality. However, the condition of homeomorphism to $S^{1}$ of a closed curve is imposed in the case of the sequential functional group embeddings as presented in the next subsection. 


\subsection{Functional Groups and Topological Embeddings}

In the previous section, the definitions related to topological decomposition and Schoenflies homeomorphism between two topological spaces are presented considering decomposed general group algebraic structure. In this section, the concept of functional group and the definitions related to the sequential embeddings of functional group structures in topological spaces are formulated.

Let $X \subset \mathcal{R}^{2}$ be a point set and $X_{S}=\left\{S_{i}: 1 \leq i \leq n\right\}$ be a family of finite number of point sets. Let $\tau_{X} \subseteq P(X)$ be a topology on $X$ generating a topological space denoted by $\left(X, \tau_{X}\right)$. Let $\left\{A_{i}: i \in I\right\} \subset \tau_{X}$ be such that $\forall i, k \in I, A_{i} \cap A_{k}=\phi$ if $i \neq k$, where $I$ is the countable index set as stated earlier. If $S_{X}=\underset{1 \leq i \leq n}{\cup} S_{i}$ is the entire collection of elements, then $l: S_{X} \rightarrow(B \subset X)$ is an injective continuous embedding. It is considered that $\forall S_{i} \in X_{S}$ the property hom $\left(S_{i}, S^{1}\right)$ is maintained indicating that embeddings are continuous closed curves in $\left(X, \tau_{X}\right)$ homeomorphic to $S^{1} \subset \mathcal{R}^{2}$. Moreover, the embeddings maintain the condition that $l\left(S_{i}\right) \cap l\left(S_{k}\right)=\phi$ whenever $i \neq k$.

The homeomorphism of embedding is maintained under restriction as given in the following definition: Let $F_{S}: S^{1} \rightarrow \mathcal{R}^{2}$ be an embedding in a topological product space. There exists a Schoenflies homeomorphism $g: \mathcal{R}^{2} \rightarrow \mathcal{R}^{2}$ such that the restriction maintains $\left.g\right|_{S^{1}}=F_{S}$.

As a natural consequence, this form of Schoenflies homeomorphism also generates separable components in topological spaces. In the following subsections, the concept of functional group structure and its sequential as well as contractible embeddings into normal topological spaces are formulated as a set of definitions.

\subsubsection{Functional Group}

Let $f_{i}: S_{i} \rightarrow S_{i}$ be a bijection. A functional group $G_{i}=\left(S_{i}, *_{i}, f_{i}\right)$ under binary and closed algebraic operation, $*_{i}: S_{i}^{2} \rightarrow S_{i}$ is defined as,

$$
\begin{aligned}
& \forall x, y \in S_{i}, x *_{i} y \in f_{i}\left(S_{i}\right), \\
& \exists x \in S_{i}: f_{i}(x)=e_{i} \in S_{i}, \\
& \forall y \in S_{i}, \exists z \in S_{i}, y *_{i} z=z *_{i} y=e_{i}, \\
& \forall y \in S_{i}, y *_{i} e_{i}=y=e_{i} *_{i} y, \\
& \forall y \in S_{i}, f_{i}(y) *_{i} f_{i}\left(e_{i}\right) \neq f_{i}(y) .
\end{aligned}
$$

It is not considered that, ${ }_{i}: S_{i}^{2} \rightarrow S_{i}$ is commutative to maintain generality. The embedding of such functional groups in the topological space is defined in the following subsection.

\subsubsection{Functional Group Embeddings}

Let $\left(X, \tau_{X}\right)$ be a topological space and $\left\langle f_{i}\right\rangle_{i=1}^{n}$ be a sequence of functions forming functional groups. The composition $\left(l \circ f_{i}\right): S_{X} \rightarrow A_{i}$ is a functional group embedding in $\left(X, \tau_{X}\right)$ if $A_{i} \in \tau_{X}$ and $\exists E_{i} \subset A_{i}$ such that $\forall x \in E_{i}$ the open subset $U_{x} \subset E_{i}$ is a neighborhood of $x$ and $\exists U_{y} \subset S_{i}$ for $y \in S_{i}$ such that $U_{y} \subseteq\left(l \circ f_{i}\right)^{-1}\left(U_{x}\right)$.

As a natural extension of the concept, the embedding maintains the following condition, $E_{i}=\left(l \circ f_{i}\right)\left(S_{X}\right)$ in $\left(X, \tau_{X}\right)$. Moreover, the embeddings form corresponding functional groups in topological subspaces in $\left(X, \tau_{X}\right)$ denoted by $\left(E_{i}, *_{i}\right)=G_{i}$ in $A_{i} \in \tau_{X}$ without imposing any notational modification of respective algebraic operation in the topological subspace. The next definition presents homeomorphisms between two embedded functional groups in the topological space $\left(X, \tau_{X}\right)$.

\subsubsection{Embedded Group Homeomorphism}

Let $\left\{A_{i}, A_{k}\right\} \subset \tau_{X}$ be such that $A_{i} \cap A_{k}=\phi$ in the topological space $\left(X, \tau_{X}\right)$. If $\left(l \circ f_{i}\right)\left(S_{X}\right)$ and $\left(l \circ f_{k}\right)\left(S_{X}\right)$ are two functional group embeddings in $E_{i} \subset A_{i}$ and $E_{k} \subset A_{k}$, then $h_{i k}: E_{i} \rightarrow E_{k}$ is a functional group homeomorphism defined on $\left(E_{i}, *_{i}\right)$ and $\left(E_{k}, *_{k}\right)$ as $\forall x, y \in E_{i}, h_{i k}\left(x *_{i} y\right)=$ $h_{i k}(x) *_{k} h_{i k}(y)$. 
Note that, the defined embedded group homeomorphism in subspaces in $\left(X, \tau_{X}\right)$ is a direct adaptation of standard group homeomorphism. The other interesting observation is that topological $\operatorname{hom}\left(E_{i}, S^{1}\right)$ and $\operatorname{hom}\left(E_{k}, S^{1}\right)$ conditions are still valid in the space $\left(X, \tau_{X}\right)$ and the monotone class property is not imposed while constructing this definition. The multiple embeddings in $\left(X, \tau_{X}\right)$ with the condition $E_{i} \cap E_{k}=\phi$ would result in the formation of Jordan curve components as defined below.

\subsubsection{Jordan Curve Components}

The Jordan curve components are defined by following Jordan curve theorem [14]. Let $\left(l \circ f_{i}\right):\left(S_{i} \subset S_{X}\right) \rightarrow A_{i}$ be an embedding in topological space $\left(X, \tau_{X}\right)$. If $\exists C_{i} \subset A_{i}$ such that $\partial C_{i}=\left(l \circ f_{i}\right)\left(S_{i}\right)$ and the component is given by $C_{i}=C_{i}^{o} \cup \partial C_{i}$, then $C_{i}$ is a generated closed component by embedding in $\left(X, \tau_{X}\right)$.

Hence, if one considers $\left\langle f_{i}\right\rangle_{i=1}^{n}$ as a finite sequence of functions along with the composition, then a sequence of embeddings would be generated in $\left(X, \tau_{X}\right)$ topological space. The definition of sequential embeddings is presented below.

\subsubsection{Sequential Embeddings}

Let $\left\langle S_{i}\right\rangle_{i=1}^{n}$ be a sequence of closed curves and $M_{i}=\left\{B_{\alpha} \subset A_{i}: A_{i} \in \tau_{X}, i, \alpha \in I\right\}$ be a monotone class. The sequence of embeddings by $\left\langle\left(l \circ f_{i}\right)\right\rangle_{i=1}^{n}$ embeds a sequence of functional groups through each $\left(l \circ f_{\alpha}\right):\left(S_{\alpha} \subset S_{X}\right) \rightarrow B_{\alpha}$ in the topological space $\left(X, \tau_{X}\right)$.

The sequence of embeddings in a topological space generates multiple components and respective component boundaries. The following definition presents component boundaries due to sequential embeddings, where the $\operatorname{hom}\left(\left(l \circ f_{i}\right)\left(S_{\alpha}\right), S^{1}\right)$ condition is maintained.

\subsubsection{Component Boundary Embeddings}

Let $C^{\otimes}=\left\langle C_{i}\right\rangle_{i=1}^{n}$ be a sequence of components embedded in topological space $\left(X, \tau_{X}\right)$ generated by $\left\langle\left(l \circ f_{i}\right)\right\rangle_{i=1}^{n}$. In the topological space $\forall C_{i}, C_{i+1} \in C^{\otimes}$ the boundary is given by,

$$
\begin{aligned}
& S_{i+1} \subset S_{X}, A_{i} \in \tau_{X}, \\
& C_{i} \subset A_{i}, B_{\alpha} \subset C_{i}, C_{i+1} \subset B_{\alpha}, \\
& \partial C_{i+1}=\left(l \circ f_{i+1}\right)\left(S_{i+1}\right) .
\end{aligned}
$$

Evidently, the set $B_{\alpha}$ is open in $\left(X, \tau_{X}\right)$ for $\alpha \in I$ and $\partial C_{i} \cap \partial C_{i+1}=\phi$. The boundaries form partitions between components in the topological space. If the topological space is considered to be Hausdorff, then a uniform contraction can be defined as given below.

\subsubsection{Uniform Contraction}

In $\left(X, \tau_{X}\right)$ topological space, if $\partial C_{i} \neq \phi$ and $\partial C_{i+k} \neq \phi$, where $k \in N, k>0$, then $\nabla: \partial C_{i} \rightarrow \partial C_{i+k}$ is called a Banach-type uniform contraction if $\forall x \in \partial C_{i+k}, \exists N_{x} \subset \partial C_{i+k}, \exists A_{\alpha} \subset \partial C_{i}$ such that $N_{x}$ is an open neighborhood of $x$ and $\forall y \in A_{\alpha}, \nabla(y) \in N_{x}$, where $A_{\alpha}$ is also open in $X$. Thus, one can view $\nabla$ as a surjective map maintaining hom $\left(\partial C_{i+k}, S^{1}\right)$ in $\left(X, \tau_{X}\right)$.

The uniform contraction is generalized in nature, indicating that the contraction can be performed for any value of $k$. The only restriction is that the contraction cannot be made into $X^{c}=\phi$ with respect to topological space $\left(X, \tau_{X}\right)$.

\section{Main Results}

This section presents the main results as a set of theorems derived from the proposed concepts, definitions and structures. The properties of functional group embeddings in normal topological spaces are formulated considering that the embeddings are homeomorphic to $S^{1}$ (i.e., they are complete with one-point compactification). Moreover, the embeddings of functional groups into a normal topological 
space are sequential in nature. In a stricter sense, the condition of formation of a monotone class is considered as a special case.

In the beginning, it is shown that, the sequences of embeddings are mutually disjoint within the normal topological space.

Theorem 1. In $C^{\otimes}=\left\langle C_{i}\right\rangle_{i=1}^{n}$ of a $\left(X, \tau_{X}\right)$ normal topological space, if $\partial C_{k} \neq \phi$ and $\partial C_{k+1} \neq \phi$, then $\exists S_{k}, S_{k+1} \in$ $\left\langle f_{i}\left(S_{i}\right)\right\rangle_{i=1}^{n}$ such that $\left(l \circ f_{k}\right)\left(S_{k}\right) \cap\left(l \circ f_{k+1}\right)\left(S_{k+1}\right)=\phi$.

Proof. Let $\left(X, \tau_{X}\right)$ be a topological space and $S^{\otimes}=\left\langle f_{i}\left(S_{i}\right)\right\rangle_{i=1}^{n}$ be a finite sequence of functional groups. Let $\left(l \circ f_{k}\right): S_{k} \rightarrow A_{k}$ be the embedding in corresponding open set $A_{k} \in \tau_{X}$, where $S_{k} \in S^{\otimes}$. If $\left\langle\left(l \circ f_{k}\right)\right\rangle_{k=1}^{n}$ is a finite sequence of embeddings in $\left(X, \tau_{X}\right)$, then the $C^{\otimes}=\left\langle C_{i}\right\rangle_{i=1}^{n}$ sequence of components will be generated in $\left(X, \tau_{X}\right)$. Let us consider that $\forall C_{k}, C_{k+1} \in C^{\otimes}, \partial C_{k} \neq \phi$ and $\partial C_{k+1} \neq \phi$ in $\left(X, \tau_{X}\right)$. This indicates that if $\left(l \circ f_{k+1}\right): S_{k+1} \rightarrow A_{k+1}$ is an embedding with $C_{k+1} \subset C_{k}$, then $A_{k+1} \subset A_{k}$, where $A_{k+1}$ is open in $\left(X, \tau_{X}\right)$ such that $\partial C_{k+1} \subset A_{k+1}$. Thus, considering that $\left(X, \tau_{X}\right)$ is a normal topological space, if $\bar{B} \subset X$ is such that $\forall k \in[1, n] \subset I, A_{k} \subset \bar{B}$, then $M_{\bar{B}}=\left\{A_{i+k}: i \in Z_{0}^{+}\right\}$is a monotone class in $\bar{B}$ if and only if $A_{k+i} \subset A_{k+j}$ whenever $i>j$. Hence, in $A_{k} \in \tau_{X}$ open subspace, it is true that $\left(l \circ f_{k+1}\right)\left(S_{k+1} \in S^{\otimes}\right)=\partial C_{k+1}$ and $\partial C_{k+1} \subset A_{k+1}$ in $\bar{B} \subset X$. Moreover, as $f_{k}\left(S_{k}\right)$ and $f_{k+1}\left(S_{k+1}\right)$ are two functional groups, so $f_{k}\left(S_{k}\right) \cap f_{k+1}\left(S_{k+1}\right)=\phi$ as they are distinct and separated. Hence, in the normal $\left(X, \tau_{X}\right)$ topological space $\left(l \circ f_{k}\right)\left(S_{k}\right) \subset A_{k}$ and $\left(l \circ f_{k+1}\right)\left(S_{k+1}\right) \subset A_{k+1}$, where $\left(l \circ f_{k}\right)\left(S_{k}\right) \cap\left(l \circ f_{k+1}\right)\left(S_{k+1}\right)=\phi$.

If one considers that a monotone class of group embeddings in a topological space is mutually disjoint, then there exists a sequence of fixed points in the set of generated components. If the embedding space is finite, then the sequence is also finite.

Theorem 2. If $\left\langle\left(l \circ f_{i}\right)\right\rangle_{i=1}^{n}$ is a sequence of disjoint functional group embeddings in finite normal topological space $\left(X, \tau_{X}\right)$, then $\left\langle x_{i}\right\rangle_{i=1}^{n}$ is a finite sequence of fixed points in $C^{\otimes}$.

Proof. Let $\left(X, \tau_{X}\right)$ be a normal topological space having Hausdorff property. If $\left(X, \tau_{X}\right)$ is finite, then $\exists\left\{A_{i}: i \in I\right\} \subset \tau_{X}$ such that $A_{i}$ is open and finite indicating that $A_{i}{ }^{c}$ is also finite (but not necessarily compact). Let $\left\langle\left(l \circ f_{i}\right)\right\rangle_{i=1}^{n}$ be a sequence of functional group embeddings in respective $A_{i}$ such that $\left(l \circ f_{i}\right)\left(S_{X}\right) \cap\left(l \circ f_{k}\right)\left(S_{X}\right)=\phi$ whenever $i \neq k$ and $n<+\infty$. Let $C^{\otimes}$ be a sequence of Jordan components generated by $\left\langle\left(l \circ f_{i}\right)\right\rangle_{i=1}^{n}$ in the corresponding $A_{i} \in \tau_{X}$. If $\left\langle\left(l \circ f_{i}\right)\right\rangle_{i=1}^{n}$ is a monotone class such that $\forall i \leq n, \partial C_{i} \subset A_{i}$, then $\underset{1 \leq i \leq n}{\cup} C_{i} \subset \underset{1 \leq i \leq n}{\cup} A_{i}$ in the topological space $\left(X, \tau_{X}\right)$, where $C_{i} \cap C_{k} \neq \phi$ if $i \leq n, k \leq n$ and $i \neq k$. Let $g: B_{i} \rightarrow B_{i}$ be a pair-wise continuous function in $\underset{1 \leq i \leq n}{\cup} A_{i} \subset X$ such that $B_{i}=C_{i} \backslash C_{i+1}^{o}$, where $\forall i \leq n, \partial C_{i} \subset C_{i}$. As $\left(\partial C_{i} \cup \partial C_{i+1}\right) \subset B_{i}$, so $B_{i}{ }^{c}$ is open in $\left(X, \tau_{X}\right)$ indicating that the function $g($.$) is bounded in every B_{i} \subset X$. Thus, $\forall C_{i} \subset A_{i}, \exists x_{i} \in B_{i}$ such that $g\left(x_{i}\right)=x_{i}$. Hence, the $\left\langle x_{i}\right\rangle_{i=1}^{n}$ is a sequence of fixed points in normal $\left(X, \tau_{X}\right)$ topological space under disjoint functional group embeddings.

Lemma 1. If $\left(X, \tau_{X}\right)$ is compact, then $\left\langle x_{i}\right\rangle_{i=1}^{+\infty}$ is convergent, where $x_{l} \in C_{l}$ is a limit.

Proof. Let $\left(X, \tau_{X}\right)$ be a compact topological space and $\left\langle\left(l \circ f_{i}\right)\right\rangle_{i=1}^{+\infty}$ be a sequence of embeddings of functional groups in $S_{X}$ into open subspace $A \in \tau_{X}$. If $\left(l \circ f_{i}\right)\left(S_{X}\right)$ and $\left(l \circ f_{k}\right)\left(S_{X}\right)$ generate two closed components in $A \in \tau_{X}$, then $C_{i}=C_{i}{ }^{o} \cup E_{i}$ and $C_{k}=C_{k}^{o} \cup E_{k}$ respectively, where $\left(l \circ f_{i}\right)\left(S_{i} \subset S_{X}\right)=E_{i}$ and $\left(l \circ f_{k}\right)\left(S_{k} \subset S_{X}\right)=E_{k}$. However, as $S_{i} \cap S_{k}=\phi$ in $S_{X}$ because the functional groups are mutually disjoint, so $E_{i} \cap E_{k}=\phi$ in $A \in \tau_{X}$. Moreover, $\exists B_{i}, B_{k} \subset X$ open sets in normal topological space such that $C_{i} \subset B_{i}, C_{k} \subset B_{k}$ and $B_{k} \subset B_{i}$ whenever $k>i$. Thus, sequence of embeddings $\left\langle\left(l \circ f_{i}\right)\right\rangle_{i=1}^{+\infty}$ generates $C^{\otimes}$ in $A \in \tau_{X}$. Again, if $\left(X, \tau_{X}\right)$ is compact, then $A^{c}$ is compact, where $\partial A \subset A^{c}$ in $\left(X, \tau_{X}\right)$. Thus, in the normal and closed topological subspace, $\cup_{1 \leq i<+\infty} E_{i} \subset \bar{A}$, where $\bar{A}=A \cup \partial A$. Hence, if $\left\langle x_{i}\right\rangle_{i=1}^{+\infty}$ is a 
sequence of fixed-points in compact $\bar{A}$, then $\left(l \circ f_{i}\right) \rightarrow C_{l}$ and $\exists x_{l} \in \partial C_{l}$ such that $x_{i} \rightarrow x_{l}$ in $\left(X, \tau_{X}\right)$. Furthermore, as $\partial C_{l} \subset C_{l}$ so $x_{l} \in C_{l}$ in $\bar{A}$.

It is noted earlier that the embeddings of functional groups are homeomorphic to $S^{1}$ in $\left(X, \tau_{X}\right)$ topological space. The neighborhood system of fixed points of the convergent sequence in embedding subspace characterizes the nature of embeddings. It also reaffirms the condition that the underlying topological space is normal.

Theorem 3. If $E_{i}$ and $E_{k}$ are functional group embeddings in normal topological space $\left(X, \tau_{X}\right)$, then the fixed points $x_{\alpha} \in C_{i}$ and $x_{\beta} \in C_{k}$ have neighborhoods such that $U_{\alpha} \cap U_{\beta}=\phi$, where $U_{\alpha} \subset E_{i}$ and $U_{\beta} \subset E_{k}$.

Proof. Let $G_{i}=\left(S_{i}, *_{i}, f_{i}\right)$ and $G_{k}=\left(S_{k},{ }^{*} k, f_{k}\right)$ be two functional groups such that $S_{i} \cap S_{k}=\phi$. Let the two corresponding embeddings be $\operatorname{hom}\left(E_{i}, S^{1}\right)$ and $\operatorname{hom}\left(E_{k}, S^{1}\right)$ in the normal topological space $\left(X, \tau_{X}\right)$. If $\left\langle x_{i}\right\rangle_{i=1}^{+\infty}$ is a convergent sequence in $A \in \tau_{X}$ and $\left(E_{i} \cup E_{k}\right) \subset A$, then $\exists C_{i}, C_{k} \in C^{\otimes}$ such that $x_{\alpha}, x_{\beta} \in\left\langle x_{i}\right\rangle_{i=1}^{+\infty}$, where $x_{\alpha} \in C_{i}$ and $x_{\beta} \in C_{k}$ are fixed points in the respective closed components in $\left(X, \tau_{X}\right)$. However, $[i<k] \Rightarrow\left[\partial C_{k} \subset C_{i}\right]$ implication is maintained in open subspace $A \in \tau_{X}$ and as $S_{i} \cap S_{k}=\phi$, so $\partial C_{i} \cap \partial C_{k}=\phi$ maintaining disjoint embedding condition. Moreover, the embeddings are homeomorphic to $S^{1}$ and generate closed components in $A \in \tau_{X}$. Thus, it is indeed true that, $x_{\alpha} \in \partial C_{i}, x_{\beta} \in \partial C_{k}$ and $\left(\partial C_{i} \cup \partial C_{k}\right) \subset A$ in the topological space $\left(X, \tau_{X}\right)$. As the topological space is normal as well as Hausdorff, hence $\exists U_{\alpha} \subset E_{i}, x_{\alpha} \in U_{\alpha}$ and $\exists U_{\beta} \subset E_{k}, x_{\beta} \in U_{\beta}$ such that $U_{\alpha} \cap U_{\beta}=\phi$, where $\left(U_{\alpha} \cup U_{\beta}\right) \subset \bar{A}$ in normal $\left(X, \tau_{X}\right)$.

Remark 2. The extension of the above property indicates that normal topological space allows normal embedded subspaces. As the topological space $\left(X, \tau_{X}\right)$ is normal, thus $\exists A_{\alpha}, A_{\beta} \subset X$ such that $U_{\alpha} \subset \overline{A_{\alpha}}$ and $U_{\beta} \subset \overline{A_{\beta}}$. Moreover, the normal subspace containing the monotone class embeddings maintains the condition given as $\partial C_{i} \subset A_{\alpha}, \partial C_{k} \subset A_{\beta}$ and $A_{\alpha} \cap A_{\beta} \neq \phi$ in $\left(X, \tau_{X}\right)$.

Interestingly, the mutual disjoint embedding of functional groups is independent of order relation in embedding sequence. This property is maintained as long as a monotone class is formed within a topological subspace. It is important to note that, when a converging sequence of fixed points are considered within a compact subspace containing embeddings, the order of embeddings is considered to be fixed according to the sequence.

Theorem 4. In the normal topological space $\left(X, \tau_{X}\right)$, if $C_{i}, C_{k} \in C^{\otimes}$ such that $|k-i| \geq 1$, then the components are not separable independent of embedding sequence.

Proof. Let $\left(X, \tau_{X}\right)$ be a normal topological space and $\exists C_{i}, C_{k} \in C^{\otimes}$ be in the subspace $A \in \tau_{X}$, where the subspace is open and $|k-i| \geq 1$. Suppose, the components are separable in $\left(X, \tau_{X}\right)$ independent of embeddings sequence in $C^{\otimes}$. Thus, if $\left(C_{i} \backslash \partial C_{i}\right) \cap C_{k}=C_{i} \cap\left(C_{k} \backslash \partial C_{k}\right)=\phi$ in $\left(X, \tau_{X}\right)$, then $\exists W, V \subset X$ open subspaces such that $C_{i} \subset W, C_{k} \subset V$ and $W \cap V=\phi$. Thus, $W$ and $V$ are separations in $\left(X, \tau_{X}\right)$. However, in this case, either $C_{i} \in X \backslash C^{\otimes}$ or $C_{k} \in X \backslash C^{\otimes}$, which is a contradiction. Thus, if $C_{i}, C_{k} \in C^{\otimes}$ is in the embedding subspace $A \in \tau_{X}$, then $(W \cup V) \subset A$ and $W \cap V \neq \phi$, where $|k-i| \geq 1$. Hence, the closure $\bar{A}$ is not a separable subspace and as a result the components are not separable or independent of embedding sequence.

Corollary 1. The uniform contraction has a role in determining separation in topological spaces. In the normal topological space $\left(X, \tau_{X}\right)$, if $C_{i}, C_{k} \in C^{\otimes}$ such that $k>i$ and $\partial C_{k}=\nabla\left(\partial C_{i}\right)$ then $\partial C_{i} \cap \partial C_{k}=\phi$. This is a relatively straight conclusion from the above-mentioned theorem. 
The continuous contraction in normal topological spaces homeomorphic to $S^{1}$ invites the requirement of surjectivity in a sequence of embeddings. However, such a sequence should form a monotone class in a normal topological subspace.

Theorem 5. In a normal topological space $\left(X, \tau_{X}\right)$, the uniform contraction $\nabla: \partial C_{i} \rightarrow \partial C_{k}$ is a surjection in $C^{\otimes}$, where $k>i$.

Proof. Let $\left(X, \tau_{X}\right)$ be a normal topological space and $\exists A \in \tau_{X}$ such that $C_{i}, C_{k} \subset A$, where $\left\{C_{i}, C_{k}\right\} \subset C^{\otimes}$. Let $\nabla: \partial C_{i} \rightarrow \partial C_{k}$ be a uniform contraction and $k>i$. Now, the components $C_{i}^{o} \subset A$ and $C_{k}^{o} \subset A$ are dense because $C_{i}=C_{i}^{o} \cup E_{i}$ and $C_{k}=C_{k}^{o} \cup E_{k}$ in $A \in \tau_{X}$, where $E_{i}, E_{k}$ are functional group embeddings in topological subspace. Moreover, as $\bar{A}=A \cup \partial A$ is not separable and $C_{i} \cup C_{k} \subset A$, thus $\left\{C_{i}, C_{k}\right\} \subset C^{\otimes}$ are not separable. Again $C_{k}^{o} \subset C_{i}^{o}$ as $k>i$ in the sequence of embeddings $C^{\otimes}$ in the normal subspace $A \in \tau_{X}$. Moreover, the sequence of embeddings maintains the homeomorphism as, $\forall C_{i} \in C^{\otimes}, \operatorname{hom}\left(\partial C_{i}, S^{1}\right)$ in $A \in \tau_{X}$. Thus, if $\nabla: \partial C_{i} \rightarrow \partial C_{k}$ is a continuous contraction, then $\forall x \in \partial C_{k}, \exists y \in \partial C_{i}$ such that $\nabla\left(N_{y} \subset \partial C_{i}\right) \subset\left(N_{x} \subset \partial C_{k}\right)$, where $N_{x}, N_{y}$ are open neighborhoods of $x, y$ respectively. Hence, the uniform contraction $\nabla: \partial C_{i} \rightarrow \partial C_{k}$ is a surjection in $C^{\otimes}$, where the topological space is normal in nature.

The surjective contraction supports the monotone class of components generated by a sequence of functional group embeddings in a normal topological subspace. The existence of Kakutani fixed points in a converging sequence in such a subspace containing embedded components invites the semicontinuity of finite set valued function within the normal subspace containing embeddings sequence. This property is presented in the next theorem.

Theorem 6. If $\alpha \in \partial C_{k}$ and $\beta \in \partial C_{i}$ with $i>k$ are two Kakutani fixed points on embeddings in a normal topological space, then there is a semicontinuous set-valued function $\gamma: \partial C_{i} \rightarrow \partial C_{k}$ such that $\gamma(\beta) \cap U_{\alpha} \neq \phi$, where $\alpha \in U_{\alpha}$ and $U_{\alpha}$ is an open set.

Proof. Let $\left(X, \tau_{X}\right)$ be a normal topological space having embedded sequence of functional groups forming a monotone class structure generated by $C^{\otimes}$. Let $\left\{C_{i}, C_{k}\right\} \subset C^{\otimes}$ be such that there are two Kakutani fixed points $\alpha \in \partial C_{k}$ and $\beta \in \partial C_{i}$ with $i>k$ in $A \in \tau_{X}$. If $\gamma: \partial C_{i} \rightarrow \partial C_{k}$ is a set-valued function, then $\exists w \in \gamma(x), \exists v \in \gamma(y)$ such that $U_{w} \cap U_{v} \neq \phi$, where $w \in U_{w}, v \in U_{v}$ are open sets in $A \in \tau_{X}$. However, if $\gamma($.$) is semicontinuous, then \forall x, y \in \partial C_{i}, \gamma(x) \cap \gamma(y)=\phi$ indicates that the mapping is unique. Moreover, in the normal subspace of embedding, $\cup \mathcal{i}_{i} \subset \bar{A}$ and the sequence of fixed points $\left\langle x_{i}\right\rangle_{i=1}^{+\infty}$ in $\bar{A}$ is a converging sequence, where $\{\alpha, \beta\} \subset\left\langle x_{i}\right\rangle_{i=1}^{+\infty}$. Thus, $\exists B \subset \partial C_{k}$ open set such that $\gamma(\beta) \subset B$. If $\alpha \in B$ then it leads to the conclusion that, $U_{\alpha} \subseteq \gamma(\beta)$. Otherwise, if $\alpha \notin B$ then $\exists D \subset \partial C_{k}$ such that $B \cap D \neq \phi$, where $\alpha \in D$. Hence, in any case, $\exists U_{\alpha} \subset X$ such that $\gamma(\beta) \cap U_{\alpha} \neq \phi$ in $\left(X, \tau_{X}\right)$.

Generally, Schoenflies homeomorphism is defined in between two separable topological spaces containing embeddings. Thus, the Schoenflies homeomorphism can exist within multiple subspaces in a normal topological space. Suppose multiple functional group embeddings are distributed within the subspaces of a normal topological space. If the functional groups are disjoint and homeomorphic, then there can be interplay between embedded group homeomorphism and Schoenflies homeomorphism. This interaction is presented as a theorem next.

Theorem 7. Let $\left(X, \tau_{X}\right)$ be a normal topological space and $\left\{A_{k}, A_{i}\right\} \subset \tau_{X}$ be such that $A_{k} \cap A_{i}=\phi$. If Schoenflies homeomorphism exists as $g_{S}: A_{k} \rightarrow A_{i}$, then there is a group homeomorphism between embedded functional groups $G_{k}=\left(S_{k}, *_{k}, f_{k}\right)$ and $G_{i}=\left(S_{i}, *_{i}, f_{i}\right)$ in $A_{k}$ and $A_{i}$, respectively. 
Proof. Let $\left(X, \tau_{X}\right)$ be a normal topological space and the two disjoint subspaces are, $\left\{A_{k}, A_{i}\right\} \subset \tau_{X}$. Let $G_{k}=\left(S_{k},{ }^{*}, f_{k}\right)$ and $G_{i}=\left(S_{i}, *_{i}, f_{i}\right)$ be two functional groups such that $S_{i} \cap S_{k}=\phi$ and $\left(S_{i} \cup S_{k}\right) \subset S_{X}$. Thus, there can be two functional group embeddings in normal $\left(X, \tau_{X}\right)$ given as, $\left(l \circ f_{k}\right): S_{X} \rightarrow A_{k}$ and $\left(l \circ f_{i}\right): S_{X} \rightarrow A_{i}$ in the respective subspaces, which are normal category of topological spaces. If the Schoenflies homeomorphism $g_{S}: A_{k} \rightarrow A_{i}$ exists in $\left(X, \tau_{X}\right)$, then $\left.g_{S}\right|_{E_{k}}=\left(l \circ f_{i}\right)\left(S_{X}\right)$. Thus, $\forall x, y \in\left(l \circ f_{k}\right)\left(S_{X}\right)$ in $A_{k}, \exists z \in\left(l \circ f_{i}\right)\left(S_{X}\right)$ such that $g_{S}\left(x *_{k} y\right)=z$ in $A_{i}$. Moreover, as $G_{k}=\left(S_{k},{ }_{k}, f_{k}\right)$ is a functional group, hence $\left(l \circ f_{k}\right)\left(x *_{k} y\right) \in A_{k}$ is maintained due to closure property of the embedded functional group. Let $h_{k i}:\left(E_{k} \subset A_{k}\right) \rightarrow\left(E_{i} \subset A_{i}\right)$ be a bijection in $\left(X, \tau_{X}\right)$. If one considers that $h_{k i}(x) *_{i} h_{k i}(y)=z \in E_{i}$ by following existing Schoenflies homeomorphism, then $\exists w \in E_{k}$ such that $h_{k i}(w)=z$ in the embedded subspace, where $x *_{k} y=w \in E_{k}$. Hence, $h_{k i}($.$) is an embedded functional$ group homeomorphism preserved by $\left.g_{S}\right|_{E_{k}}=\left(l \circ f_{i}\right)\left(S_{X}\right)$.

Remark 3. From the above theorem, it can be further concluded that $\operatorname{hom}\left(E_{k}, S^{1}\right) \Rightarrow \operatorname{hom}\left(E_{i}, S^{1}\right)$ in the normal topological space $\left(X, \tau_{X}\right)$ and the embeddings are not contractible in nature. As a result, the sequences of homeomorphic functional group embeddings are not in a monotone class.

The above observation can be extended under uniform contraction between two homeomorphic embedding spaces, which are separable. The group homeomorphism exists under uniform contraction if and only if the embedding spaces maintain Schoenflies homeomorphism in separable subspaces as given in the next theorem.

Theorem 8. Let $\left(X, \tau_{X}\right)$ be a normal topological space and $\left\{A_{k}, A_{i}\right\} \subset \tau_{X}$ be such that $A_{k} \cap A_{i}=\phi$, If $\left\{\left(l \circ f_{a}\right)\left(S_{X}\right),\left(l \circ f_{b}\right)\left(S_{X}\right)\right\} \subset A_{i}$ and $\left\{\left(l \circ f_{c}\right)\left(S_{X}\right),\left(l \circ f_{d}\right)\left(S_{X}\right)\right\} \subset A_{k}$ are embeddings in respective subspaces, such that $h_{a c}: E_{a} \rightarrow E_{c}$ and $h_{b d}: E_{b} \rightarrow E_{d}$ are group homeomorphisms, then there are uniform contractions $\nabla: \partial C_{a} \rightarrow \partial C_{b}$ and $\nabla: \partial C_{c} \rightarrow \partial C_{d}$ if Schoenflies homeomorphism exists as, $g_{S}: A_{i} \rightarrow A_{k}$.

Proof. Let $\left(X, \tau_{X}\right)$ be a normal topological space and $\left\{A_{k}, A_{i}\right\} \subset \tau_{X}$ be such that $A_{k} \cap A_{i}=\phi$ and $\Lambda=\left\{G_{a}, G_{b}, G_{c}, G_{d}\right\}$ be a set of disjoint functional groups. Let $\left\{\left(l \circ f_{a}\right)\left(S_{X}\right),\left(l \circ f_{b}\right)\left(S_{X}\right)\right\} \subset A_{i}$ and $\left\{\left(l \circ f_{c}\right)\left(S_{X}\right),\left(l \circ f_{d}\right)\left(S_{X}\right)\right\} \subset A_{k}$ be the respective embeddings in corresponding subspaces such that $E_{a} \cap E_{b}=\phi$ and $E_{c} \cap E_{d}=\phi$ in $\left(X, \tau_{X}\right)$. Let $g_{S}: A_{i} \rightarrow A_{k}$ be a Schoenflies homeomorphism such that $\left.g_{S}\right|_{E_{a}}=\left(l \circ f_{c}\right)\left(S_{X}\right)$ and $\left.g_{S}\right|_{E_{b}}=\left(l \circ f_{d}\right)\left(S_{X}\right)$. Thus, the functional group embeddings and Schoenflies homeomorphism will generate components in subspaces as $C_{a}, C_{b} \subset A_{i}$ and $C_{c}, C_{d} \subset A_{k}$. If $\nabla: \partial C_{a} \rightarrow \partial C_{b}$ is a uniform contraction, then $\forall x, y \in \partial C_{b}, \exists U_{x}, U_{y} \subset \partial C_{a}$ such that $\nabla\left(\alpha_{x} \in U_{x}\right)=$ $x$ and $\nabla\left(\beta_{y} \in U_{y}\right)=y$. Suppose $h_{a c}:\left(E_{a} \subset A_{i}\right) \rightarrow\left(E_{c} \subset A_{k}\right)$ and $h_{b d}:\left(E_{b} \subset A_{i}\right) \rightarrow\left(E_{d} \subset A_{k}\right)$ are embedded functional groups' homeomorphisms in normal topological space, $\left(X, \tau_{X}\right)$. Thus, within the homeomorphic group embedding subspace, $h_{b d}\left(x *_{b} y\right) \in E_{d}$ and $h_{b d}\left(x *_{b} y\right)=h_{b d}(x) *_{d} h_{b d}(y)$, where $G_{b}=\left(S_{b}, *_{b}, f_{b}\right), G_{d}=\left(S_{d}, *_{d}, f_{d}\right)$ are respective functional groups. Similarly, $h_{a c}\left(u *_{a} v\right) \in E_{c}$ and $h_{a c}\left(u *_{a} v\right)=h_{a c}(u) *_{c} h_{a c}(v)$, where $G_{a}=\left(S_{a}, *_{a}, f_{a}\right), G_{c}=\left(S_{c}, *_{c}, f_{c}\right)$ are respective functional groups and $\{u, v\} \subset\left(l \circ f_{a}\right)\left(S_{a} \subset S_{X}\right)$. Hence, there exists a uniform contraction, $\nabla: \partial C_{c} \rightarrow \partial C_{d}$ in $A_{k}$ such that $\forall m, n, \in \partial C_{d}, \exists U_{m}, U_{n} \subset \partial C_{c}$ such that $\nabla\left(\alpha_{m} \in U_{m}\right)=m$ and $\nabla\left(\beta_{n} \in U_{n}\right)=n$, where $h_{a c}\left(U_{u}\right) \subset U_{m}$ and $h_{a c}\left(U_{v}\right) \subset U_{n}$.

This indicates that there is a strong isometry between two separable normal topological subspaces containing functional group embeddings if the disjoint embedded functional groups are homeomorphic to each other. Moreover, in this case, the Schoenflies homeomorphism exists between the subspaces.

\section{Comparative Analysis}

This section presents the detailed comparative analysis of different constructions of group structures, decompositions and associated varieties of topological embeddings. Traditionally, the general group algebraic structures are discrete and do not impose the continuity as well as finiteness criteria. The concept of continuity in a group structure as well as algebraic operation is 
incorporated in topological groups, which are in a special category. The similarity between topological groups and the proposed algebraic construction of functional groups is that both structures incorporate continuity within the generated group structures. However, the distinctions of the proposed algebraic construction of functional groups are that: (1) the algebraic construction is generalized in nature; (2) they are compactly generated; and (3) they are sequentially embeddable with isometry within the normal topological space. The sequential embeddings of functional groups within a topological space generate a monotone class of components. Interestingly, the embeddings of topologically decomposed generalized group structures do not allow contraction. However, the proposed sequential embeddings of functional groups homeomorphic to $S^{1}$ support Banach-type contractive embeddings within the normal spaces. The similarity between the embeddings of the decomposed generalized group structure and the sequential embeddings of the functional group structure is that both varieties are complete with one-point compactification within a topological space. The parametric comparison between the two categories of structures is presented in Table 1.

Table 1. Comparison between decomposable general group and functional group structures.

\begin{tabular}{cccccc}
\hline $\begin{array}{c}\text { Group } \\
\text { Structures }\end{array}$ & Homeomorphism & $\begin{array}{c}\text { Topological } \\
\text { Decomposability }\end{array}$ & $\begin{array}{c}\text { Sequentially } \\
\text { Embeddable }\end{array}$ & Contraction & $\begin{array}{c}\text { One-point } \\
\text { Compactification }\end{array}$ \\
\hline$G_{i}=\left(S_{i}, *_{i}, f_{i}\right)$ & $S^{1}$ & Yes & Yes & Yes & Possible \\
$G_{X}=(X, * X)$ & $G_{Y}=(Y, * Y)$ & Yes & No & No & Possible \\
\hline
\end{tabular}

Traditionally, there are two broad categories of decomposable group constructions, namely direct product groups and semidirect product groups. Although the traditional decompositions of generalized group algebraic structures are based on the existence of Cartesian products of corresponding subgroups, in the case of topological decomposition of general group structures, this condition for the existence of independent subgroups is relaxed. The uniqueness property of decompositions of generalized group structures varies between semidirect product and direct product. However, the topological decomposition relaxes this requirement further. The topological decomposition of general group algebraic structures and the sequentially embeddable functional group structures are unique in nature. The comparison of properties related to group decompositions and embeddings related to varieties of groups is summarized in Table 2 .

Table 2. Comparison between various group decomposition and embedding structures.

\begin{tabular}{cccccc}
\hline Decomposition/Embeddings & Uniqueness & $\begin{array}{c}\text { Global } \\
\text { Continuity }\end{array}$ & $\begin{array}{c}\text { Local } \\
\text { Continuity }\end{array}$ & Identity & Separability \\
\hline Semidirect product & No & Yes & Yes & Shared & No \\
\hline Direct product & Yes & No & Yes & Distinct & Yes \\
\hline Topological decomposition & Yes & No & Yes & Distinct & Yes \\
\hline Functional group embeddings & Yes & No & Yes & Distinct & $\begin{array}{c}\text { Monotone class } \\
\text { components: No, } \\
\text { otherwise: Yes }\end{array}$ \\
\hline
\end{tabular}

It is interesting to note that the local continuity property is maintained by every decomposition and topological embedding types. However, the global continuity property is maintained by the semidirect product. This results in the categorization of decomposition with respect to the separability property in regard to the generated components. The semidirect product is globally continuous having a shared identity, hence the decomposed components are not topologically separable in nature. However, the topological decomposition of general group structures can be locally continuous in nature and separable. Furthermore, the sequential embeddings of functional groups in a normal topological space are component wise separable if and only if they are not in a monotone class of isometry in the embedded subspaces. 


\section{Conclusions}

The embeddable topological decompositions of general group algebraic structures and the functional group embeddings maintain the property of one-point compactification. The proposed algebraic constructions of functional groups are generalized in nature generating compact groups. The constructed functional groups are homeomorphic to circle groups and are embeddable within a normal topological space in a sequence. The embeddings support Banach-type contraction in underlying Hausdorff space. The contraction generates a monotone class of sequential embeddings following the Schoenflies variety. The functional group homeomorphism between two spaces is successfully preserved within the respective monotone classes under uniform contraction. Moreover, there exists a compact subspace within the normal topological space containing the sequential embeddings of functional groups. Interestingly, the sequential embeddings of functional groups in-line with Schoenflies variety may not generate multiple compact and separable components if it forms a monotone class within the normal topological space. Otherwise, the two sequential topological embeddings in disjoint subspaces are separable. The proposed topological embeddings of functional groups support inclusion of Kakutani fixed points and the embedding subspace is sequentially complete in nature.

Funding: This research was funded by Gyeongsang National University, Jinju, ROK.

Acknowledgments: The author would like to thank the editors and anonymous reviewers for their helpful comments and suggestions.

Conflicts of Interest: The author declares no conflict of interest.

\section{References}

1. Tall, F.D. More topological applications of generic elementary embeddings. In Topology and Its Applications; Elsevier: Amsterdam, The Netherlands, 1992; Volume 44, pp. 353-358.

2. Pedersen, E.K. Embeddings of topological manifolds. Ill. J. Math. 1975, 19, 440-447. [CrossRef]

3. Robb, A.; Teicher, M. Applications of braid group techniques to the decomposition of moduli spaces, new examples. In Topology and Its Applications; Elsevier: Amsterdam, The Netherlands, 1997; Volume 78, pp. 143-151.

4. Wolfram, T.; Ellialtioglu, S. Applications of Group Theory to Atoms, Molecules and Solids; Cambridge University Press: Cambridge, UK, 2014; ISBN 978-1-107-02852-4.

5. Daugulis, P. Nonuniqueness of semidirect decompositions for semidirect products with directly decomposable factors and applications for dihedral groups. Algebra Discret. Math. 2017, 23, 204-215.

6. Linckelmann, M. The Block Theory of Finite Group Algebras; Cambridge University Press: Cambridge, UK, 2018; Volume 2.

7. Loebl, E.M. Group Theory and Its Applications; Academic Press Inc.: New York, NY, USA, 1975; Volume 3, pp. 107-108.

8. Becher, V.; Grigorieff, S. Borel and hausdorff hierarchies in topological spaces of choquet games and their effectivization. Math. Struct. Comp. Sci. 2015, 25, 1490-1519. [CrossRef]

9. Xiao, Z.; Sanchez, I.; Tkachenko, M. Topological groups whose closed subgroups are separable, and the product operation. In Topology and Its Applications; Elsevier: Amsterdam, The Netherlands, 2019; Volume 259.

10. Joseph, J. On H-closed and minimal hausdorff spaces. Proc. Am. Math. Soc. 1976, 60, 159-170.

11. Biasi, C.; Daccach, J.; Saeki, O. A primary onstruction to topological embeddings and its applications. In Manuscripta Mathematica; Springer: Berlin/Heidelberg, Germany, 2001; Volume 104, pp. 97-110.

12. Guggenheimer, H. The jordan and schoenflies theorems in axiomatic geometry. Am. Math. Mon. 1978, 9, 85 . [CrossRef]

13. McCord, M.C. Embedding P-like compacta in manifolds. Can. J. Math. 1967, 19, 321-332. [CrossRef]

14. Tverberg, H. A proof of the jordan curve theorem. Bull. Lond. Math. Soc. 1980, 12, 34-38. [CrossRef]

15. Little, C.H.C.; Vince, A. Embedding schemes and the jordan curve theorem. In Topics in Combinatorics and Graph Theory; Physica-Verlag: Heidelberg, Germany, 1990. 
16. Hollings, C. Embedding semigroups in groups: Not as simple as it might seam. Arch. Hist. Exact Sci. 2014, 68, 641-692. [CrossRef]

17. Petrashen, M.I.; Trifonov, E.D. Applications of Group Theory in Quantum Mechanics; Dover Publications Inc.: New York, NY, USA, 2009; ISBN 978-0-486-47223-2.

18. Bagchi, S. Projective and non-projective varieties of topological decomposition of groups with embeddings. Symmetry 2020, 12, 450. [CrossRef]

19. Aragona, R.; Caranti, A. The group generated by the round functions of a GOST-like cipher. Ann. Math. Pur. Appl. 2017, 196, 1-17. [CrossRef]

20. Santo, R.D.; Dikranjan, D.; Bruno, A.G. Characterized subgroups of the circle group. Ric. Math. 2018, 67, 625-655. [CrossRef]

21. Vasco, M.I.G.; Steinwandt, R. Group Theoretic Cryptography; Chapman \& Hall/CRC: Boca Raton, FL, USA, 2015; ISBN 9781584888369.

22. Chen, B.; Du, C.Y.; Wang, R. The groupoid structure of groupoid morphisms. J. Geom. Phys. 2019, 145, 103486. [CrossRef]

23. Montgomery, D. What is a topological group? Am. Math. Mon. 1945, 52, 302-307. [CrossRef]

24. Hales, T.C. The jordan curve theorem- formally and informally. Am. Math. Mon. 2007, 114, 882-894. [CrossRef]

25. Weiss, W. Partitioning topological spaces. In Mathematics of Ramsey Theory; Springer: Berlin/Heidelberg, Germany, 1990; Volume 5, pp. 154-171.

26. Bourbaki, N. A Panorama of Pure Mathematics; Academic Press: Waltham, MA, USA, 1982; ISBN 0-12-215560-2.

(C) 2020 by the author. Licensee MDPI, Basel, Switzerland. This article is an open access article distributed under the terms and conditions of the Creative Commons Attribution (CC BY) license (http://creativecommons.org/licenses/by/4.0/). 